\title{
Effect of Bacillus coagulans Unique IS-2 in Inflammatory Bowel Disease (IBD): A Randomized Controlled Trial
}

\author{
V Deepak Bamola ${ }^{1}$, Divya Dubey ${ }^{1}$, Projoyita Samanta ${ }^{1}$, Saurabh Kedia ${ }^{2}$, Ratna Sudha \\ Madempudi $^{3}$, Jayanthi Neelamraju ${ }^{3}$, Vineet Ahuja ${ }^{2}$, Rama Chaudhry ${ }^{1 *}$ \\ ${ }^{1}$ Department of Microbiology, ${ }^{2}$ Department of Gastroenterology, \\ All India Institute of Medical Sciences, New Delhi, India \\ ${ }^{3}$ Centre for Research \& Development, Unique Biotech Ltd., Plot No. 2, Phase-II, \\ Alexandria Knowledge Park, Hyderabad, Telangana, 500078, India \\ *Corresponding author
}

\begin{abstract}
Probiotic intervention is an important approach for the treatment and health restoration in inflammatory bowel disease (IBD). A study was conducted to assess the effect of Bacillus coagulans Unique IS-2 in IBD patients. Recruited subjects were given either probiotic $B$. coagulans Unique IS-2 or placebo for 4 weeks as per randomization. Survival of the given probiotic strain in GI, presence of beneficial gut bacteria, serum cytokines, serum serotonin and serum dopamine, symptoms of disease, physical, behavioral and psychological parameters of the subjects were evaluated before and after intervention. In this study $B$. coagulans Unique IS-2 was well tolerated with no severe adverse events in IBD patients and demonstrated good survival in GI tract by significantly high detection in probiotic treated group ( $\mathrm{p}<0.001$ ). Significant enhancement in beneficial Lactobacilli was observed in probiotic treated group $(\mathrm{p}<0.01)$. Metagenomic analysis also showed an increase in the abundance of bacterial genera Bacillus, Lactobacillus, Bifidobacterium, Faecalibacterium, Bacteroides, Megamonas, Lachnospira, Blautia, Alistipes and decrease in Sutterella, Dialister, Roseburia and Megasphaera was observed in the post intervention samples in the treatment group. Increased secretion of cytokine IL-10 and variable decrease in the secretion of IL-6, IL-1 $\beta$, TNF- $\alpha$, IL -17 and IL -23 was observed in in the probiotic treated group. Post intervention change in serum serotonin and serum dopamine was not significant in both the groups. A reduction in the severity of disease symptoms and improvement in the physical, behavioral and psychological parameter was observed in the probiotic treated group. The observed results demonstrated that B. coagulans Unique IS-2 with SMT was effective in adult IBD patients. Study was registered with Clinical Trials Registry India (CTRI) - (registration no.- CTRI/2019/11/022087).
\end{abstract}

Keywords: Inflammatory bowel disease (IBD), Gut Microbiota, Probiotic, Bacillus coagulans, Cytokines, Randomized Controlled Trial (RCT) 


\section{Introduction}

Inflammatory bowel disease (IBD) is a gastrointestinal (GI) disease characterized by chronic inflammation. The incidence of IBD is rising all over the world ${ }^{1}$ including Asia $^{2}$. Across the globe India is projected to have one of the highest IBD burden in spite of having lower prevalence as compared to the $\mathrm{West}^{3,4}$. IBD significantly diminishes the quality of life of the affected individuals by disturbing health, daily work, education, and social relationships which cause a considerable impact on health and economy ${ }^{5}$. Etiology and pathogenesis of IBD is multi-factorial and involves genetics, ethnicity, diet, lifestyle, environmental factors, immunity and gut microbiota of the individual ${ }^{6,7}$. Higher stress level and disturbance in psychology in IBD patients may trigger the inflammation and increases the severity of disease ${ }^{8}$.

The human gut microbiota has demonstrated physiological functions associated with homeostasis, nutrition, immunity and defense of the host. Normally the gut maintains a homeostatic condition but in IBD this homeostasis gets disturbed and leads to intestinal inflammation ${ }^{9}$. Down-regulation of the immune responses may allow the damaged site to heal and reset the normal physiological functions ${ }^{7}$. Alteration in the gut microbiota, referred to as dysbiosis, plays a key role in the pathogenesis of $\mathrm{IBD}^{10,11}$. Dysbiosis may occur in both inflamed and non-inflamed areas in patients ${ }^{12}$ and is well recognized sign of IBD $^{13}$. Modification of gut microbiota with probiotic intervention to attenuate inflammatory activity and prevent relapses in IBD are considered as an important approach for treatment and health restoration in $\mathrm{IBD}^{11,14}$ by relieving intestinal dysbiosis and GI inflammation ${ }^{10}$.

GI disorders modulate the gut function and influence the emotional and cognitive factors including mood, anxiety, pain and negative effects, decision making, restlessness. This change in emotional and cognitive factors may be associated with serum serotonin and dopamine in IBD patients ${ }^{15}$. The lowering of serotonin levels can result in mood disorders, such as anxiety or depression and hence consider playing an important role in regulating physical and psychological symptoms ${ }^{16}$ and activation of immune response and gut inflammation $^{15}$. Dopamine is an important neurotransmitter which affects behavior, psychology, immune functions and GI functions and its unregulated production in IBD is already reported ${ }^{17}$. Evaluation of these factors has turn out to be an important factor to monitor the severity of the disease. Gut microbiota and probiotics have demonstrated the effect on behavior, psychology, mood, and cognition ${ }^{18,19}$. 
74 Bacillus strains are stable at room temperature and hence gaining a lot of attention. $B$.

75 coagulans is a spore-forming, gram-positive, non-pathogenic, facultative anaerobic, lactic

76 acid-producing bacteria and resistant to high temperatures ${ }^{20}$. B. coagulans Qualified

77 Presumption of Safety (QPS) $\operatorname{list}^{21}$ and has been reported Generally Recognized as Safe

78 (GRAS) and considered safe by European Union Food Safety Authority (EFSA) and US

79 Food and Drug Administration (FDA). B coagulans Unique IS-2 (ATCC PTA-11748,

80 MTCC 5260) is a shelf-stable, resistant to bile acids and acidic conditions of the stomach,

81 clinically established probiotic strain and with proven safety and efficacy in the treatment of

82 constipation, diarrhea ${ }^{22-25}$ bacterial vaginosis ${ }^{26}$ and irritable bowel syndrome (IBS) ${ }^{27}$. In this

83 study we assessed the effect of $B$ coagulans Unique IS-2 on adults with inflammatory bowel

84 disease (IBD) receiving standard medical treatment (SMT). Survival of the given probiotic $B$

85 coagulans Unique IS2 in the gut, presence of beneficial gut bacteria, serum cytokines,

86 serotonin and dopamine, IBD symptoms, physical and psychological parameters were

87 evaluated before and after intervention. 


\section{Methodology}

- Study design: Randomized, double blind, placebo controlled trial. Study was registered with Clinical Trials Registry (CTRI) India (CTRI/2019/11/022087).

- Site: Outpatients from a tertiary care hospital.

- Ethical approval and written informed consent: Ethical approval for the study was obtained from Institute Ethics Committee of AIIMS, New Delhi, India (Ref IEC.478/07.10.2016.OP-7). Expected duration of participation, expected benefits, associated risks, and maintenance of confidentiality of records were explained to each participant and a written informed consent was obtained before enrollment in the study.

- Subject / Selection of patients: Clinically diagnosed adult patients of ulcerative colitis (UC) with mild to moderate severity between age group (18-60 years) under standard Medical treatment (SMT) patients were included. Simple Clinical Colitis Activity Index (SCCAI) score ${ }^{28}$ was used to quantify UC disease activity. SCCAI score was calculated by evaluating different disease symptoms including bowel frequency, urgency of defecation, blood in stool, abdominal cramps and general wellbeing of the patients. SMT for the enrolled patient in this study was 5-aminosalicylic acid (5-ASA) - Sulfasalazine (3 grams/ day) or Mesalamine $800 \mathrm{mg}$ orally 3 times a day.

- Inclusion criteria: (a) Adult patient clinically diagnosed with Ulcerative Colitis (UC), (b) patients of either sex of the age range from 18-60 year, (d) patient ready to participate in the study and giving written consent, (e) patient visiting to Out Patient Department (OPD) of AIIMS for treatment.

- Exclusion criteria: (a) Patient diagnosed with any kind of carcinoma, (b) Patient diagnosed with any other gastrointestinal disease, (c) Patient suffering from Immunodeficiency disorder, (d) Patient is taking any probiotic drug/ or having consumed probiotic in the last one month, (e) patient not taking food through oral route, (f) Patient having undergone any kind of gastrointestinal surgery in the last three month.

- Enrolment of patients: After establishing the eligibility on screening, a total of 100 patients were recruited and randomized. Patients were called for baseline visit (day 0). Medical history, medications, physical examination and vital signs were assessed during hospital visit. B. coagulans Unique IS-2 (2billion-CFU/capsule) twice in day (total 4 billion CFU / per day) or placebo (matching in size and appearance, contained only excipient, maltodextrin) twice in a day was given to qualified patients for 4 weeks followed by observation and telephonic follow-up of dose compliance. 
- Sample size determination: Statistical software STATA (Version 14, USA) was used for sample calculation. To identify presence of proportion difference the assumption was made that minimum of 118 subjects required to be screened and 94 patients required assessing the endpoint in the study which will reject the null hypothesis.

- Intervention: Fully characterized FDA / DCGI/ FSSAI approved probiotic strain Bacillus coagulans Unique IS-2 (ATCC PTA-11748, MTCC 5260) was used as an intervention agent in this study for 4 weeks along with placebo as per randomization. The patients were explained to consume one capsule post meal twice a day for 4 weeks along with the SMT. The compliance was ensured by telephonic follow-up and scheduled hospital visits.

- Randomization: Computerized permuted blocks randomization was done in 1:1 ratio and generated by nQuery clinical trial design platform / Sample Size Software. It consisted of two phases: screening, baseline visit 1 (week 0 / day 0$)$, visit 2 ( 1 week after completing intervention / week 5). The randomization codes were kept blinded.

- Outcome measures: The efficacy outcomes were measured after administration by (i) detection of Bacillus coagulans Unique IS-2, (ii) Change in beneficial Lactobacillus and other gut microbiota, (iii) Change in pro and anti-inflammatory cytokines, (iv) Change in symptoms of the disease, (v) Change in serum concentration of serotonin and dopamine, (vi) Changes in physical and psychological parameters. Each participant was requested to answer the designed questionnaire for the assessment of physical, behavioral and psychological parameters as per the Hopkins Symptom Checklist (HSCL): A self-report symptom inventory ${ }^{29,30}$. Physical parameters including muscle stiffness, heartburn, headache, shakiness, sleep problem, difficulty in completing work, procrastination, overwhelming, feeling of depression, trouble relaxing, nervousness, poor concentration, restlessness and quick temper were evaluated. Parameters were evaluated based on scores, the decrease in the score indicating the reduction in the severity of symptoms and increase in the score indicating an augmentation in the severity of the symptom.

- Safety evaluation: Safety of investigational product was assessed by adverse event reporting. During hospital visit of the patient physical examination, monitoring of vital signs and routine laboratory investigations.

- Sample collection and processing: A stool sample in a sterile container and a blood sample in a plain vial were collected from each enrolled subject before and after intervention. Stool samples were aliquoted and processed for microbial identification and bacterial DNA isolation. Blood samples were processed for serum separation for cytokine assays, serotonin and dopamine concentration. 
- Microbial culture and identification: B coagulans Unique IS-2 and Lactobacillus spp. were checked in each patient before and after intervention using different bacterial media. Mueller Hinton $(\mathrm{MH})$ broth and agar (Difco Laboratory, Detroit, MI) was used for the cultivation of Bacillus strains. Sample were incubated for 24 hours at $37^{\circ} \mathrm{C}$ in $\mathrm{MH}$ broth and then plated on MH agar plate. de Man, Rogosa and Sharpe (MRS) broth and agar (Difco Laboratory, Detroit, MI) was used to grow Lactobacillus. The stool sample were incubated for 48 hours at $37^{\circ} \mathrm{C}$ in MRS broth in Anaerobic Glove Box (Anaerobic Workstation-Whitley DG250-DonWhitley Scientific, United Kingdom) in anaerobic condition and then plated on MRS agar plate. The isolated colonies were identified by standard culture and biochemical method, matrix-assisted laser desorption / ionization(MALDI-and the mass analyzer is time-of-flight (TOF) analyzer (bioMérieuxInc, USA) and molecular method.

- Molecular identification of Bacteria: B. coagulans was identified via $16 \mathrm{~S}$ rRNA sequencing using published primers: forward 5'-ACAGGGCTTTCAGATACCCG-3' and reverse 5'-CGGGGATCCGTCCATCAAAA-3'. Sequence similarity was checked using BLAST, NCBI and it was $96 \%$ identical. A known strain of B. coagulans Unique IS-2 was used as a positive control. The reaction mixture consisted of $0.5 \mu l$ of $\mathrm{dNTP}(10 \mathrm{mM})$, $0.5 \mathrm{ul}$ of DNA template $(177 \mathrm{ng} / \mu \mathrm{l}), 2.5 \mu \mathrm{l}$ of reaction buffer $(10 \mathrm{X})$ with MgCL2, $0.5 \mathrm{ul}$ of each of primers $(\mathrm{pm} / \mu \mathrm{l}), 0.5 \mu \mathrm{l}$ of $5 \mathrm{U} / \mu \mathrm{lTaq}$ DNA polymerase (Thermo Scientific, USA) and $20 \mu \mathrm{l}$ of nuclease free $\mathrm{H}_{2} \mathrm{O}$. Denaturation was done at $94^{\circ} \mathrm{C}$ for $5 \mathrm{~min}$, followed by 30 cycles consisting of, $94^{\circ} \mathrm{C}$ for $1 \mathrm{~min}, 56^{\circ} \mathrm{C}$ for $1 \mathrm{~min}$, and $72^{\circ} \mathrm{C}$ for $1 \mathrm{~min}$ which has been ended by a final amplification step at $72^{\circ} \mathrm{C}$ for $8 \mathrm{~min}$, using the PCR machine (Applied Biosystems, USA). PCR product was analyzed by the electrophoresis in $1 \%$ agarose gel and gel bands were observed and recorded using via Gel Doc System (BioRad, USA).

\section{Next generation sequencing (NGS) of Gut microbiota-}

- Fecal samples collection and DNA isolation: Fecal samples were collected in sterile container and processed for DNA isolation. Total DNA was extracted by using QIAamp DNA Stool Mini Kit (Qiagen) with some modification to increase DNA yield ${ }^{31}$. The quality and quantity of DNA was checked by Nanodrop (TECAN Nano quant). 16S rRNA amplicon sequencing was performed using Illumina MiSeq ${ }^{\circledR}$ sequencing system (Illumina, San Diego, CA, USA).

- Sequencing Methodology: Bacterial 16S rRNA hyper variable regions V3-V4 were amplified using V3-V4F (CCTACGGGNGGCWGCAG) and V3-V4R (GACTACHVGGGTATCTAATCC) primers. 25ng of DNA was used for PCR 
190

191

192

193

200

201 amplification using KAPA HiFi HotStart Ready Mix. The PCR was performed with standard protocol and the amplicons were purified using Ampure beads to remove unused primers. The amplicon product was amplified with Illumina primers to generate sequencing libraries. Qubit dsDNA assay kit was used for libraries preparation. Sequencing was done using Illumina Miseq with 2x300PE sequencing kit. The sequence data quality was checked using FastQC and MultiQC software.

- Data Analysis: The analysis was done as per standard methodology ${ }^{32}$. Only QC passed reads were transferred into mothur and the pairs were aligned. The ambiguous contigs were rejected and duplicates were merged. Chimeric sequences were identified by a known reference and UCHIME algorithm was used. Using Silva v.132 database final filtered contigs were classified into taxonomical outlines and clustered into Operational Taxonomic Unit (OTUs) and abundance was calculated. Alpha diversity was assessed for richness and relative abundance of bacteria. Chaol and ACE indices were used for richness and Shannon, Simpson, and Fisher were used for both richness and relative abundance. Kruskal-Wallis rank sum test was carried out to identify statistically significant difference among OTUs abundance between groups.

\section{- ELISA for Cytokines, serum serotonin and dopamine:}

Serum samples were tested and quantified for IL10, IL6, IL17, IL23, IL 1 $\beta$, TNF, serum serotonin and dopamine as per standard protocol and manufacture's instruction (Fine Test, Fine Biotech Co. Ltd). In brief, standards and test samples were added to 96 well plates and incubated at $37^{\circ} \mathrm{C}$ for $90 \mathrm{~min}$. After incubation, wells were washed with wash buffer and secondary antibody were added and incubated at $37^{\circ} \mathrm{C}$ for $60 \mathrm{~min}$. Further, wells were washed and HRP-Streptavidin was added and incubated at $37^{\circ} \mathrm{C}$ for $30 \mathrm{~min}$. Multiple washing was done with the wash buffer to wash unbound conjugates. TMB substrates were used to visualize HRP enzymatic reaction. Absorbance at $450 \mathrm{~nm}$ has been measured using the microplate reader (Nanodrop, Nanoquant Infinite M 200 Pro (Texan, Austria $\mathrm{GmbH}$ ) and the concentration were calculated.

- Data analysis: Here we have reported the analysis of data of the patients of ulcerative colitis (UC). Data from 48 subjects in treatment group and 49 subjects in placebo group were analysed. Statistical analysis was done by STATA statistical software (Version 14, USA). Statistical evaluation of parameters was assessed by Chi-square / two sample $t$-test and $\mathrm{p}$ value $<0.05$ was considered as statistically significant. 
234

235

236

237

238

239

240

241

242

243

244

245

246

247

248

249

250

251

252

253

254

255

256

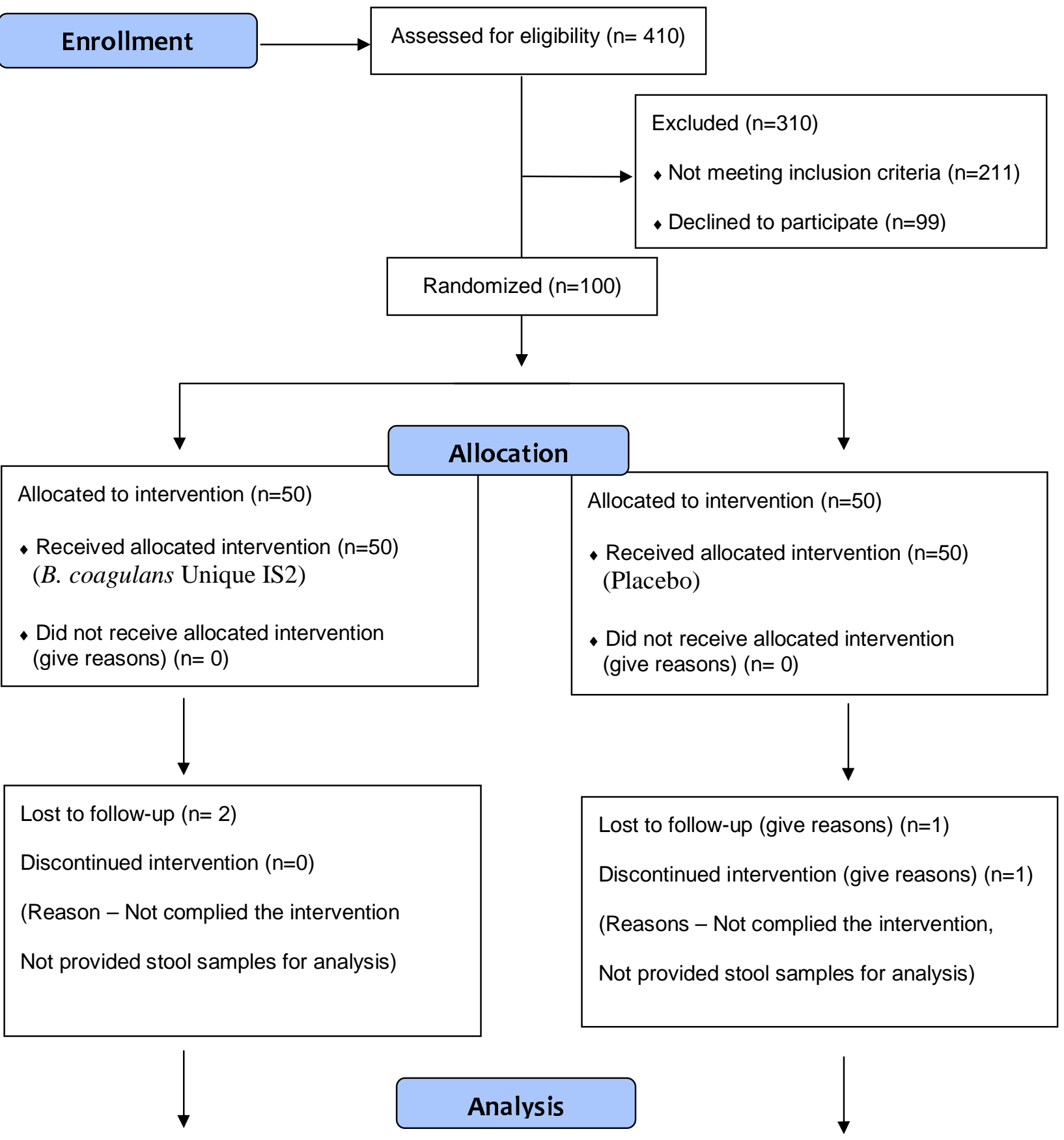

Analysed $(n=48)$

- Excluded from analysis (give reasons) $(n=2)$

Discontinued intervention

Analysed $(n=49)$

- Excluded from analysis (give reasons) $(n=1)$

Discontinued intervention 


\section{Results}

\section{Survival of Bacillus coagulans Unique IS2 in the gut}

The samples of the enrolled subjects in the both treatment and placebo group were assessed for the presence of Bacillus coagulans Unique IS-2 before and after treatment. No $B$ coagulans was detected in pre-treatment sample in both the groups. In the probiotic treated group B coagulans was detected in $65.21 \%$ subjects by microbial culture method and 76.08 $\%$ subjects by molecular method which was significant as compared to placebo $(\mathrm{p}<0.0001)$. The samples from the subjects of placebo group were also assessed for the presence of $B$ coagulans with no $B$ coagulans being detected in placebo group by both microbial and molecular methods. These results indicate that the probiotic strain $B$ coagulans Unique IS-2 was able to survive in GI tract of recruited IBD patients.

\section{Detection of beneficial Lactobacilli by microbial culture method}

The samples of the enrolled IBD patients in both probiotic treated group and placebo group were assessed for the presence of beneficial Lactobacilli, before and after intervention. Results revealed that in the probiotic treated group, beneficial Lactobacilli were detected in $54.34 \%$ and $78.26 \%$ patients before and after intervention respectively. In the placebo group beneficial Lactobacilli were detected in $52.08 \%$ and $47.91 \%$ of patients before and after intervention respectively. The detection of beneficial Lactobacilli after intervention in the probiotic treated group was significantly high $(\mathrm{p}<0.01)$ which indicated that the probiotic strain Bacillus coagulans Unique IS-2 was able to enhance the presence of beneficial Lactobacilli in recruited IBD patients.

\section{Next generation Sequencing (NGS) and Metagenomic analysis of gut microbiota}

Phyla: Operational Taxonomic Unit (OTUs) were calculated for different bacterial taxon including phyla, class, orders, families, genera and species before and after intervention in both probiotic treated and placebo group. Analysis revealed phylum Firmicutes Bacteriodetes, Proteobacteria, Actinobacteria, Euryarchaeota and Verrucomicrobia were abundant in both the groups. The average \% OTUs of phylum Firmicutes were in $33.13 \%$ and $36.23 \%$ in pre and post intervention sample respectively in treatment group and $37.90 \%$ and $42.89 \%$ in placebo group. The average \% OTUs of phylum Bacteriodetes were in $40.12 \%$ and $40.28 \%$ in pre and post intervention sample respectively in treatment group and $32.49 \%$ and $40.28 \%$ in pre and post intervention sample respectively in placebo group. OTUs of phylum Bacteriodetes were significantly increased in post intervention sample in 
medRxiv preprint doi: https://doi.org/10.1101/2021.07.18.21260556; this version posted October 30, 2021. The copyright holder for this preprint (which was not certified by peer review) is the author/funder, who has granted medRxiv a license to display the preprint in perpetuity.

It is made available under a CC-BY-NC-ND 4.0 International license .

292 placebo group. The average \% OTUs of phylum Proteobacteria were in $12.90 \%$ and $7.50 \%$

293 in pre and post intervention sample respectively in treatment group and $16.75 \%$ and $6.21 \%$ in 294 placebo group. The average \% OTUs of phylum Actinobacteria were in $6.64 \%$ and $4.29 \%$ in 295 pre and post intervention sample respectively in treatment group and $8.62 \%$ and $2.04 \%$ in 296 placebo group. Decrease in OTUs of phylum Proteobacteria and phylum Actinobacteria 297 was significant in the treatment group. The average \% OTUs of phylum Euryarchaeota were 298 in $1.02 \%$ and $2.36 \%$ in pre and post intervention sample respectively in treatment group and $2.03 \%$ and $0.55 \%$ in placebo group. OTUs of phylum Euryarchaeota were increased in post intervention sample in treatment group and decreased in placebo group. The average \% OTUs of phylum Verrucomicrobia were in $0.01 \%$ and $0.04 \%$ in pre and post intervention sample respectively in treatment group and $0.96 \%$ and $0.10 \%$ in placebo group. OTUs of phylum Verrucomicrobia were increased in post intervention sample in treatment group and decreased in placebo group.

305 Figure 1: Abundance of major bacterial phyla

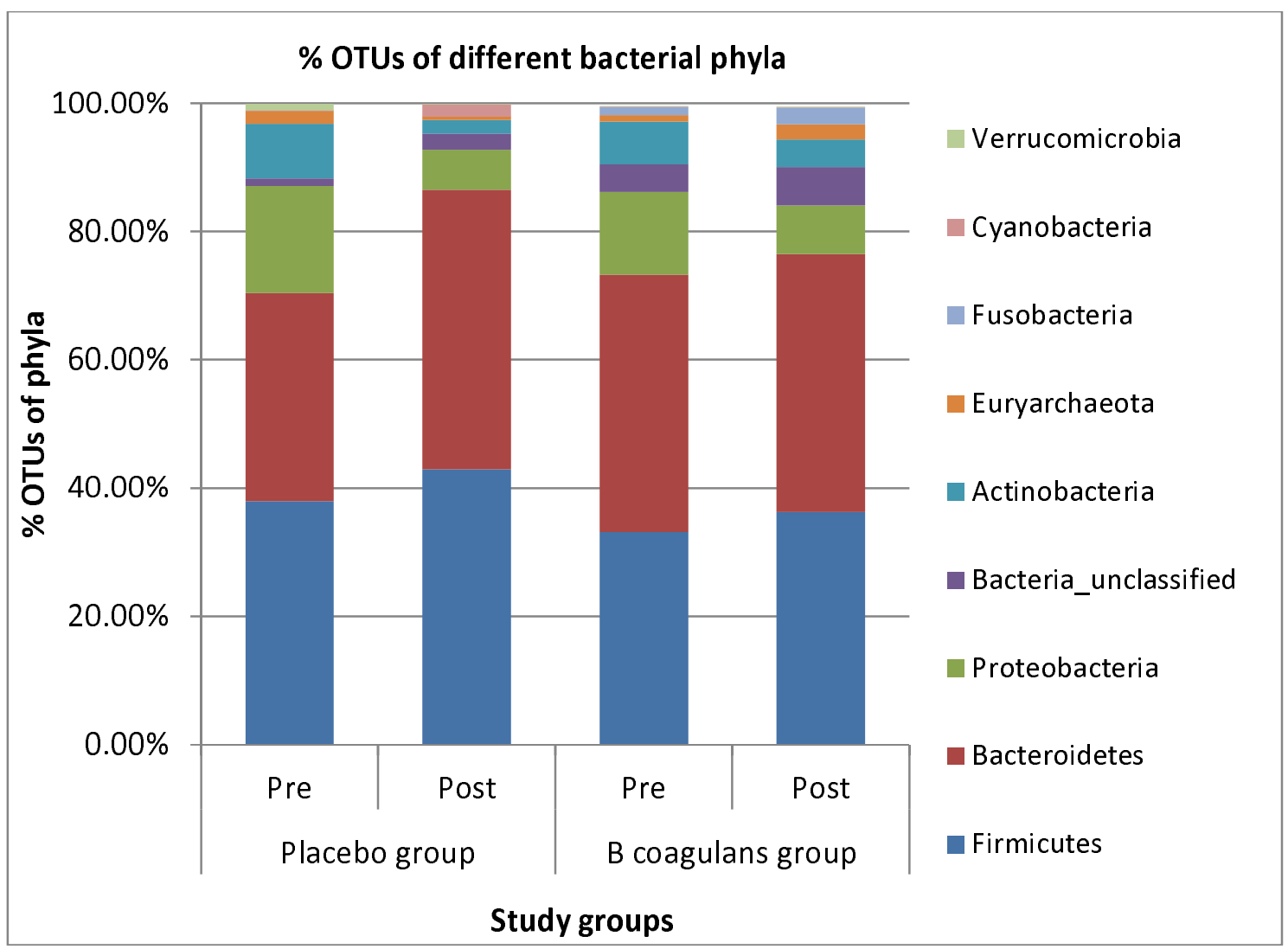

Class: The average \% OTUs of class Bacteroidia were $40.11 \%$ and $40.27 \%$ in pre and post intervention sample respectively in treatment group and $32.48 \%$ and $43.63 \%$ pre and post intervention sample respectively in placebo group. An increase in OTUs of class Bacteroidia 
medRxiv preprint doi: https://doi.org/10.1101/2021.07.18.21260556; this version posted October 30, 2021. The copyright holder for this preprint (which was not certified by peer review) is the author/funder, who has granted medRxiv a license to display the preprint in perpetuity.

It is made available under a CC-BY-NC-ND 4.0 International license.

310 was significant in the placebo group. The average \% OTUs of class Clostridia were $15.20 \%$ 311 and $22.09 \%$ in pre and post intervention sample respectively in treatment group and $20.79 \%$ and $28.82 \%$ in pre and post intervention sample respectively in placebo group. The average $\%$ OTUs of class Gammaproteobacteria were $12.87 \%$ and $7.41 \%$ in pre and post intervention sample respectively in treatment group and $16.72 \%$ and $6.16 \%$ respectively in placebo group. The average \% OTUs of class Negativicutes were $7.96 \%$ and $6.35 \%$ in pre and post intervention sample respectively in treatment group and $3.49 \%$ and $7.22 \%$ respectively in placebo group. A decrease was observed in OTUs of class Negativicutes in post intervention samples of treatment group and an increase in post intervention samples in placebo group. The average \% OTUs of class Methanobacteria were $1.02 \%$ and $2.36 \%$ in pre and post intervention sample respectively in treatment group and $2.03 \%$ and $0.55 \%$ in placebo group. An increase in OTUs of class Methanobacteria in post intervention samples was observed in treatment group and decrease in OTUs of class Methanobacteria in placebo group. The average \% OTUs of class Actinobacteria were $3.49 \%$ and $1.81 \%$ in pre and post intervention sample respectively in treatment group and $7.76 \%$ and $1.64 \%$ pre and post intervention sample respectively in placebo group.

Figure 2: Abundance of major bacterial classes

\section{\% OTUs of different bacterial classes}

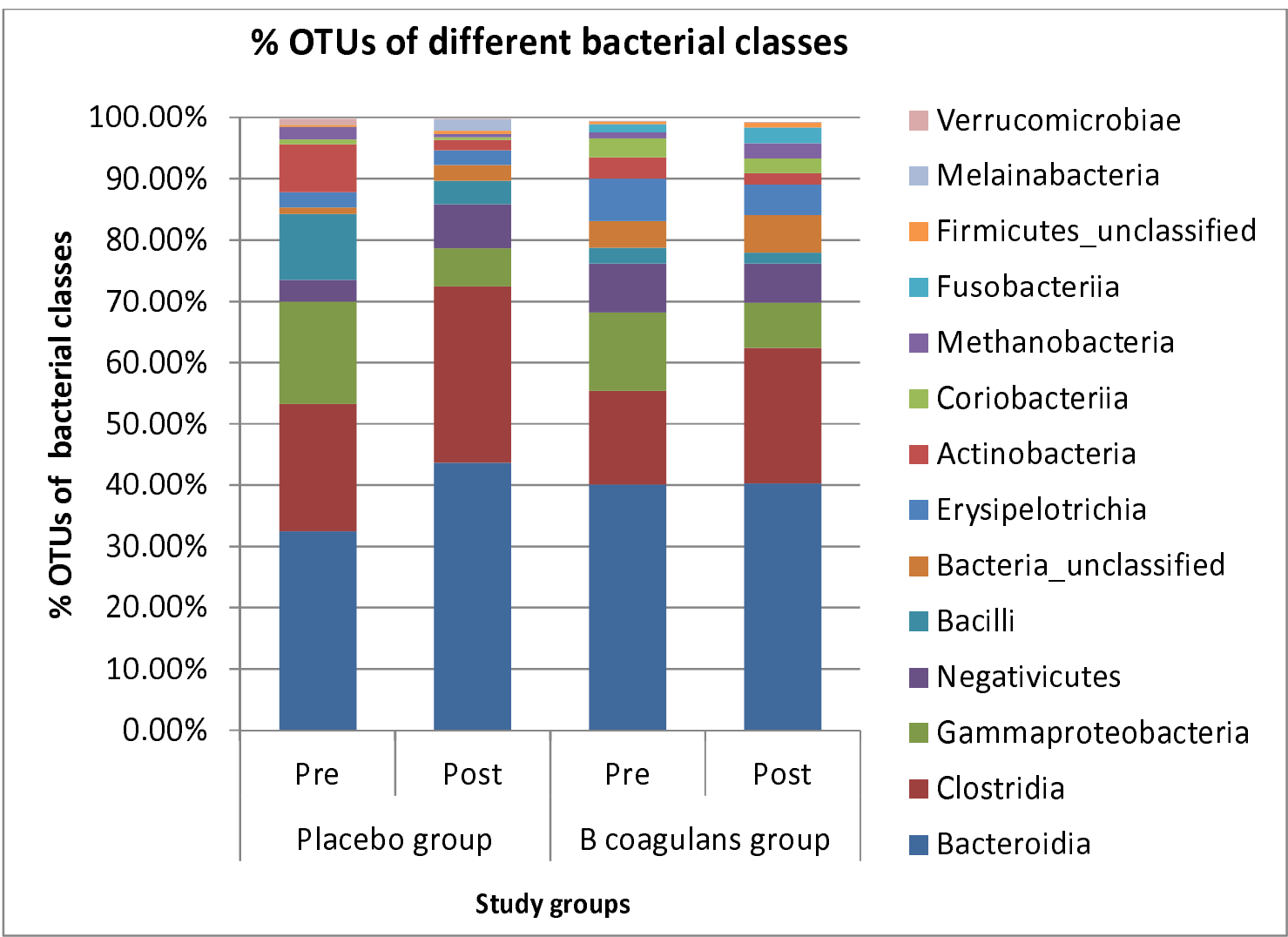


328 Order: The average \% OTUs of Bacteroidales were $39.94 \%$ and $40.03 \%$ in pre and post 329 intervention sample respectively in treatment group and $32.37 \%$ and $43.46 \%$ in placebo

330 group. A significant increase in OTUs of order Bacteroidales in post intervention samples 331 was observed in placebo group. The average \% OTUs of order Clostridiales was 15.19\% 332 and $22.08 \%$ in pre and post intervention sample respectively in treatment group and $20.79 \%$ 333 and $28.81 \%$ in placebo group. The average \% OTUs of order Selenomonadales was $8.02 \%$ 334 and $6.30 \%$ in pre and post intervention sample respectively in treatment group and 3.49\% 335 and $7.22 \%$ in placebo group. A decrease in OTUs of order Selenomonadales in post 336 intervention samples in treatment group and increase in post intervention samples in placebo 337 group. The average \% OTUs of order Lactobacillales was $1.67 \%$ and $2.49 \%$ in pre and post 338 intervention sample respectively in treatment group and $10.66 \%$ and $3.82 \%$ in placebo group. 339 An increase in OTUs of order Lactobacillales was observed in post intervention samples in 340 treatment group and decrease in post intervention samples in placebo group. The average \% 341 OTUs of order Erysipelotrichales was $7.00 \%$ and $5.03 \%$ in pre and post intervention sample 342 respectively in treatment group and $2.48 \%$ and $2.48 \%$ in placebo group. A decrease in 343 OTUs of order Erysipelotrichales in post intervention samples in treatment group and 344 increase in placebo group were observed. The average \% OTUs of order Bifidobacteriales 345 were $1.74 \%$ and $3.55 \%$ in pre and post intervention sample respectively in treatment group 346 and $7.71 \%$ and $1.61 \%$ respectively in placebo group. An increase in OTUs of order 347 Bifidobacteriales in post intervention samples in treatment group and decrease in placebo 348 group were observed.

349 Figure 3: Abundance of major bacterial orders 


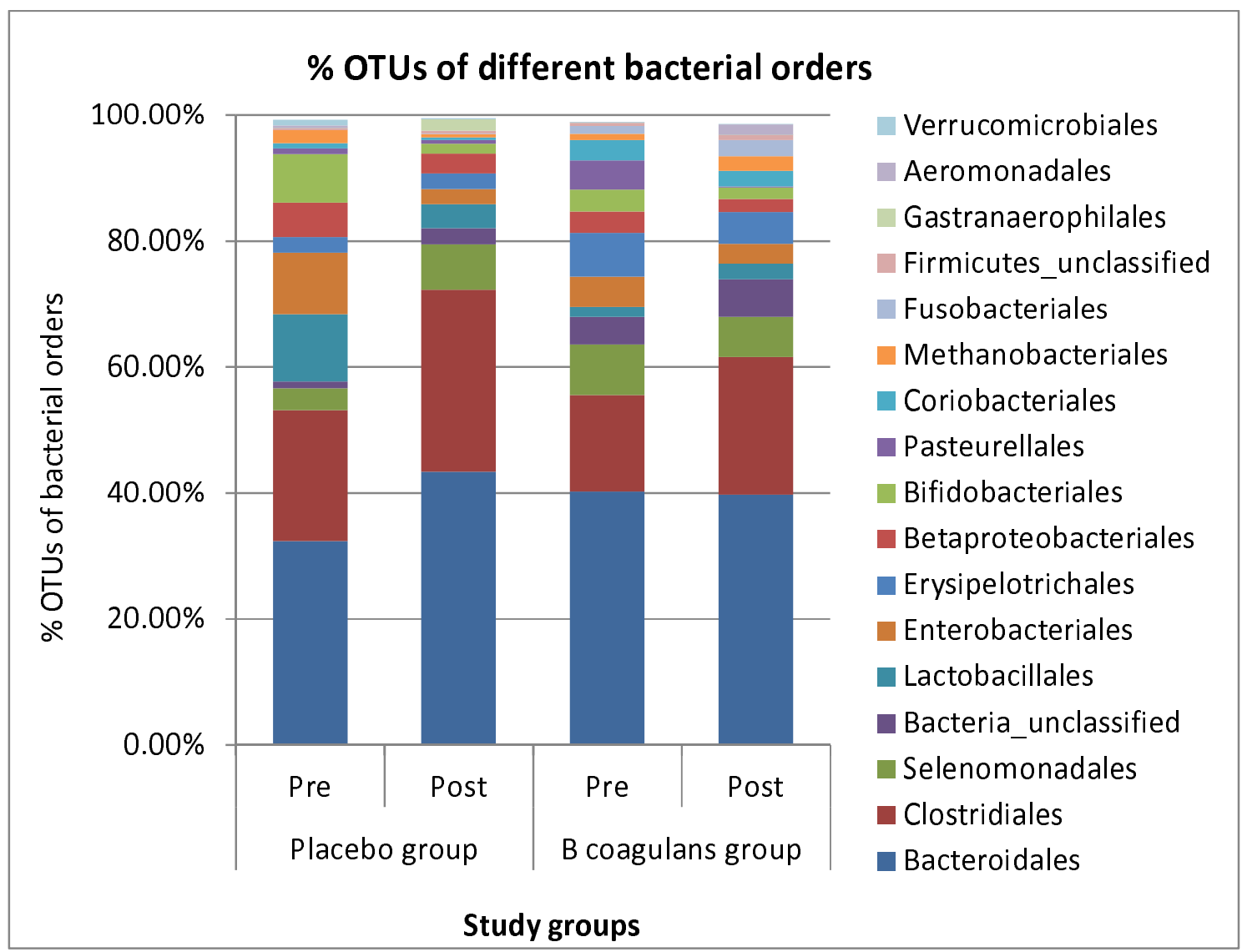

351 Family: The average \% OTUs of family Prevotellaceae was $32.66 \%$ and $33.20 \%$ in pre and post intervention sample respectively in treatment group and $8.62 \%$ and $31.96 \%$ in placebo group. Significant increase was observed in OTUs of family Prevotellaceae in post intervention samples in placebo group. The average \% OTUs of family Ruminococcaceae was $8.22 \%$ and $11.99 \%$ in pre and post intervention sample respectively in treatment group and $12.34 \%$ and $12.23 \%$ in placebo group. Increase in OTUs of family Ruminococcaceae in post intervention samples in treatment group and decrease in placebo group. The average $\%$ OTUs of family Bacteroidaceae was $4.09 \%$ and $4.58 \%$ in pre and post intervention sample respectively in treatment group $22.85 \%$ and $9.44 \%$ in placebo group. Increase in OTUs of family Bacteroidaceae in post intervention samples in treatment group and decrease in placebo group were observed. The average \% OTUs of family Lachnospiraceae was $6.14 \%$ and $8.40 \%$ in pre and post intervention sample respectively in treatment group and $6.79 \%$ and $13.48 \%$ respectively in placebo group. The average \% OTUs of family Veillonellaceae was $7.47 \%$ and $5.72 \%$ in pre and post intervention sample respectively in treatment group and $3.49 \%$ and $7.15 \%$ in placebo group. Decrease in OTUs of family Veillonellaceae in post intervention samples in treatment group and increase in placebo group were observed. The 
average \% OTUs of family Enterobacteriaceae was $4.69 \%$ and $3.14 \%$ in pre and post intervention sample respectively in treatment group and $9.79 \%$ and $2.41 \%$ respectively in placebo group. A significant decrease was observed in OTUs of family Enterobacteriaceae in post intervention samples in placebo group. The average \% OTUs of family Lactobacillaceae was $1.12 \%$ and $2.03 \%$ in pre and post intervention sample respectively in treatment group and $6.36 \%$ and $3.22 \%$ respectively in placebo group. Increase in OTUs of family Lactobacillaceae in post intervention samples in treatment group and decrease in placebo group were observed. The average \% OTUs of family Bifidobacteriaceae was $1.74 \%$ and $3.55 \%$ in pre and post intervention sample respectively in treatment group and $7.71 \%$ and $1.61 \%$ respectively in placebo group. Increase in OTUs of family Bifidobacteriaceae in post intervention samples in treatment group and decrease in placebo group were observed.

\section{Figure 4: Abundance of major bacterial families}

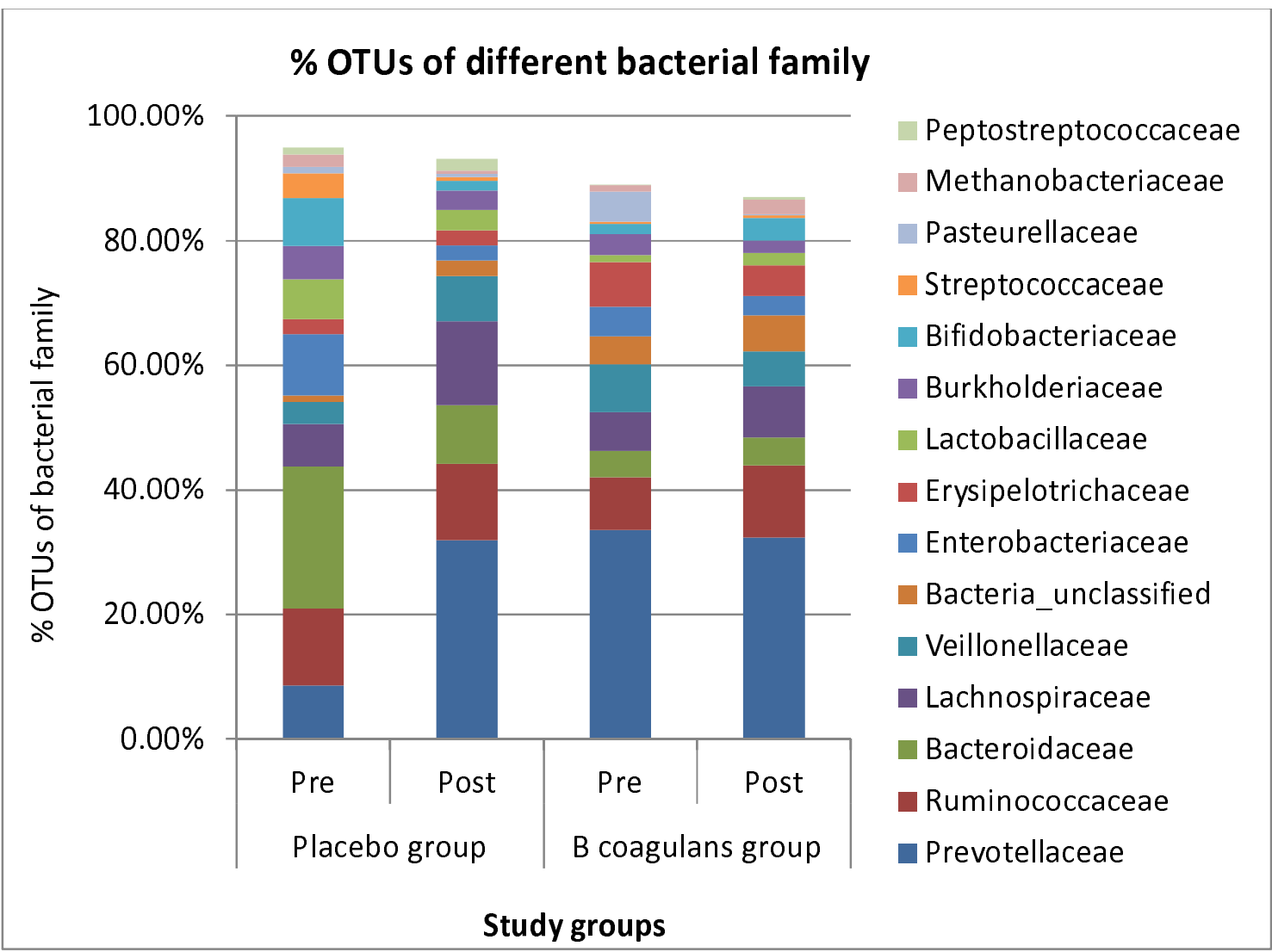

Genera: The average \% OTUs of genus Prevotella were $26.37 \%$ and $22.10 \%$ in pre and post intervention sample respectively in treatment group and $10.28 \%$ and $25.57 \%$ respectively in placebo group. Decrease in OTUs of genus Prevotella in post intervention samples in treatment group and an increase in placebo group was observed. The average \% OTUs of 
genus Bacteroides were $4.88 \%$ and $6.13 \%$ in pre and post intervention sample respectively in treatment group and $19.96 \%$ and $7.89 \%$ respectively in placebo group. An increase in OTUs of genus Bacteroides in post intervention samples in treatment group and decrease in placebo group were observed. Average \% OTUs of genus Faecalibacterium were $6.56 \%$ and $6.87 \%$ in pre and post intervention sample respectively in treatment group and $9.23 \%$ and $8.01 \%$ respectively in placebo group. An increase in OTUs of genus Faecalibacterium in post intervention samples in treatment group and decrease in placebo group were observed. The average \% OTUs of genus Lactobacillus were $1.21 \%$ and $2.59 \%$ in pre and post intervention sample respectively in treatment group and $7.33 \%$ and $3.49 \%$ respectively in placebo group. An increase in OTUs of genus Lactobacillus in post intervention samples in treatment group and decrease in placebo group were observed. The average \% OTUs of genus Bifidobacterium were $1.67 \%$ and $4.44 \%$ in pre and post intervention sample respectively in treatment group and $6.03 \%$ and $1.42 \%$ respectively in placebo group. An increase in OTUs of genus Bifidobacterium in post intervention samples in treatment group and decrease in placebo group were observed. The average \% OTUs of genus Bacillus were $0.01 \%$ and $0.04 \%$ in pre and post intervention sample respectively in treatment group and $0.001 \%$ and $0.001 \%$ respectively in placebo group. OTUs of Genus Bacillus in post intervention samples in treatment group were high while OTUs of genus Bacillus in placebo group were very low. The average \% OTUs of genus Escherichia were $3.61 \%$ and $1.24 \%$ in pre and post intervention sample respectively in treatment group and $11.06 \%$ and $1.81 \%$ in placebo group. The average \% OTUs of genus Sutterella were $3.05 \%$ and $2.22 \%$ in pre and post intervention sample respectively in treatment group and $3.60 \%$ and $1.68 \%$ in placebo group. The average $\%$ OTUs of genus Dialister were $3.37 \%$ and $1.98 \%$ in pre and post intervention sample respectively in treatment group and $0.09 \%$ and $3.66 \%$ respectively in placebo group. A decrease in OTUs of genus Dialister was observed in post intervention samples in treatment group and increase in placebo group. The average \% OTUs of genus Megamonas were $1.39 \%$ and $2.17 \%$ in pre and post intervention sample respectively in treatment group and $0.73 \%$ and $0.001 \%$ respectively in placebo group. An increase in OTUs of genus Megamonas was observed in post intervention samples in treatment group and decrease in placebo group. The average \% OTUs of genus Roseburia were $0.82 \%$ and $0.45 \%$ in pre and post intervention sample respectively in treatment group and $1.18 \%$ and $2.46 \%$ in placebo group. A decrease in OTUs of genus Roseburia was observed in post intervention samples in treatment group and increase in placebo group. The average \% OTUs of genus Megasphaera were $2.37 \%$ and $0.36 \%$ in pre and post intervention sample respectively in treatment group 
419

420

421

422

423

424

425

426

427

428

429

430

431

432

and $2.02 \%$ and $1.97 \%$ in placebo group. A decrease in OTUs of genus Megasphaera was observed in post intervention samples in both treatment and placebo group. The average \% OTUs of genus Lachnospira were $0.42 \%$ and $0.34 \%$ in pre and post intervention sample respectively in treatment group and $1.49 \%$ and $2.83 \%$ in placebo group. An increase in OTUs of genus Lachnospira was observed in post intervention samples in treatment group and decrease in placebo group. The average \% OTUs of genus Blautia were $0.25 \%$ and $0.40 \%$ in pre and post intervention sample respectively in treatment group and $0.12 \%$ and $0.25 \%$ in placebo group. An increase in OTUs of genus Blautia was observed in post intervention samples in both treatment and placebo group. The average \% OTUs of genus Alistipes were $0.08 \%$ and $0.37 \%$ in pre and post intervention sample respectively in treatment group and $0.12 \%$ and $0.04 \%$ in placebo group. An increase in OTUs of genus Alistipes in post intervention samples in treatment group and decrease in placebo group were observed.

\section{Figure 5: Abundance of major bacterial genera}

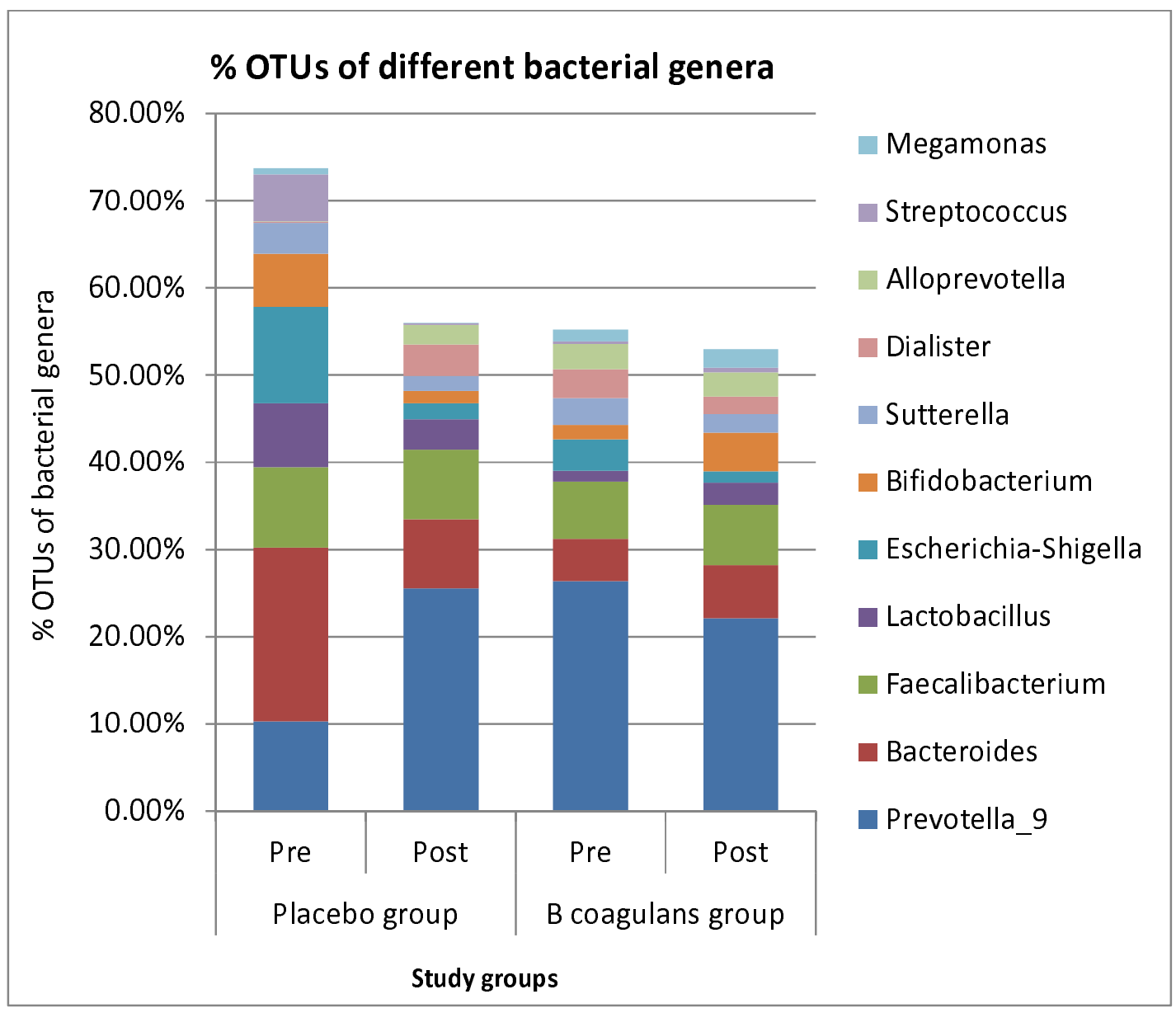


434

435

436

\section{Cytokines levels}

Serum concentrations of different cytokines were assessed before and after intervention and results are expressed as Mean $\pm \mathrm{SD}$. In the probiotic treated group serum levels of IL10 were $3.23 \pm 1.22 \mathrm{pg} / \mathrm{ml}$ and $9.25 \pm 2.6 \mathrm{pg} / \mathrm{ml}$ before and after intervention respectively with the difference being significant $(\mathrm{p}<0.05)$. In the placebo group serum IL10 levels were $2.47 \pm 1.19 \mathrm{pg} / \mathrm{ml}$ and $2.97 \pm 1.67 \mathrm{pg} / \mathrm{ml}$ before and after intervention respectively, this difference was not significant $(\mathrm{p}=\mathrm{ns})$. Results indicated that the secretion of IL-10 in IBD-UC patients was increased in $B$ coagulans Unique IS2 group. In the treatment group serum IL6 levels were $34.13 \pm 6.8 \mathrm{pg} / \mathrm{ml}$ and $17.64 \pm 5.2 \mathrm{pg} / \mathrm{ml}$ before and after intervention respectively with the difference being significant $(\mathrm{p}<0.05)$. In the placebo group serum IL6 levels were $35.85 \pm 7.6 \mathrm{pg} / \mathrm{ml}$ and $28.49 \pm 6.4 \mathrm{pg} / \mathrm{ml}$ before and after intervention respectively and this difference was not significant $(\mathrm{p}=\mathrm{ns})$. The observed results indicated that the secretion of IL-6 in IBD-UC patients was decreased in $B$ coagulans Unique IS2 group. In the probiotic treated group, serum IL17 levels were $42.82 \pm 6.9 \mathrm{pg} / \mathrm{ml}$ and $28.57 \pm 5.4 \mathrm{pg} / \mathrm{ml}$ before and after intervention respectively with the difference being significant ( $\mathrm{p}<0.05$ ). In the placebo group serum IL17 levels were $36.73 \pm 13.73 \mathrm{pg} / \mathrm{ml}$ and $33.96 \pm 14.74 \mathrm{pg} / \mathrm{ml}$ before and after intervention respectively with the difference not being significant $(\mathrm{p}=\mathrm{ns})$. The observed results indicated that the secretion of serum IL-17 was decreased in IBD-UC patients in the probiotic group. In the probiotic treated group serum IL23 levels were $938.39 \pm 56.57 \mathrm{pg} / \mathrm{ml}$ and $842.20 \pm 69.43 \mathrm{pg} / \mathrm{ml}$ before and after intervention respectively with the difference not being significant. In the placebo group serum IL23 levels were $951.59 \pm 61.31 \mathrm{pg} / \mathrm{ml}$ and $932.02 \pm 43.30 \mathrm{pg} / \mathrm{ml}$ before and after intervention respectively with the difference not being significant $(\mathrm{p}=\mathrm{ns})$.

In the treatment group serum IL-1 $\beta$ levels were $358.58 \pm 39.29 \mathrm{pg} / \mathrm{ml}$ and $267.28 \pm$ $33.88 \mathrm{pg} / \mathrm{ml}$ before and after intervention respectively with the difference being significant ( $\mathrm{p}$ $<0.05)$. In the placebo group serum IL-1 $\beta$ levels were $363.93 \pm 35.57 \mathrm{pg} / \mathrm{ml}$ and $382.59 \pm$ $37.50 \mathrm{pg} / \mathrm{ml}$ before and after intervention respectively with the difference not being significant $(\mathrm{p}=\mathrm{ns})$. The observed results indicated that the secretion of serum IL-1 $\beta$ in IBDUC patients was increased in $B$ coagulans Unique IS2 group. In the treatment group serum TNF- $\alpha$ levels were $80.33 \pm 13.68 \mathrm{pg} / \mathrm{ml}$ and $69.16 \pm 14.79 \mathrm{pg} / \mathrm{ml}$ before and after intervention respectively with the difference not significant whereas in the placebo group serum TNF- $\alpha$ levels were $76.35 \pm 14.72 \mathrm{pg} / \mathrm{ml}$ and $79.39 \pm 13.8 \mathrm{pg} / \mathrm{ml}$ before and after intervention respectively with the difference not being significant. 


\section{9}

480

Serum serotonin and dopamine levels:

In the treatment group serum serotonin level were $121.48 \pm 15.52 \mathrm{ng} / \mathrm{ml}$ and $111.30 \pm$ $17.74 \mathrm{ng} / \mathrm{ml}$ before and after intervention and the difference was not significant. In the placebo group serotonin level were $118.12 \pm 19.25 \mathrm{ng} / \mathrm{ml}$ and $109.88 \pm 11.23 \mathrm{ng} / \mathrm{ml}$ before and after intervention and the difference was not significant. In the treatment group serum dopamine level were $8.51 \pm 2.52 \mathrm{pg} / \mathrm{ml}$ and $11.74 \pm 2.25 \mathrm{pg} / \mathrm{ml}$ before and after intervention and the difference was not significant. In the placebo group dopamine level were $8.48 \pm 2.89$ $\mathrm{pg} / \mathrm{ml}$ and $10.89 \pm 2.99 \mathrm{pg} / \mathrm{ml}$, before and after intervention and the difference was not significant. There were changes in serotonin and dopamine levels in the subjects before and after intervention but the difference was not significant.

Table 1: Serum Cytokines, serotonin and dopamine levels in pre and post intervention samples in treatment and placebo group

\begin{tabular}{|c|c|c|c|c|c|c|}
\hline \multirow{2}{*}{$\begin{array}{c}\text { Cytokine } \\
(\mathrm{pg} / \mathrm{ml})\end{array}$} & \multicolumn{3}{|c|}{ Bacillus coagulans Unique IS2 group } & \multicolumn{3}{|c|}{ Placebo group } \\
\hline & Pre & Post & P value & Pre & Post & P value \\
\hline IL-10 & $3.23 \pm 1.22$ & $9.25 \pm 2.6$ & $<0.05$ & $2.47 \pm 1.19$ & $2.97 \pm 1.67$ & $\mathrm{NS}$ \\
\hline IL-6 & $34.13 \pm 6.8$ & $17.64 \pm 5.2$ & $<0.05$ & $35.85 \pm 7.6$ & $28.49 \pm 6.4$ & NS \\
\hline IL-17 & $42.82 \pm 6.9$ & $28.57 \pm 5.4$ & $<0.05$ & $36.73 \pm 13.7$ & $33.96 \pm 14.74$ & NS \\
\hline IL-23 & $938.39 \pm 56.5$ & $842.20 \pm 69.4$ & NS & $951.59 \pm 61.3$ & $932.02 \pm 43.30$ & NS \\
\hline IL-1 $\beta$ & $358.58 \pm 39.2$ & $267.28 \pm 33.8$ & $<0.05$ & $363.93 \pm 35.5$ & $382.59 \pm 37.50$ & NS \\
\hline TNF- $\alpha$ & $80.33 \pm 13.6$ & $69.16 \pm 14.7$ & NS & $76.35 \pm 14.7$ & $79.39 \pm 13.8$ & $\mathrm{NS}$ \\
\hline $\begin{array}{l}\text { Serotonin } \\
(\mathrm{ng} / \mathrm{ml})\end{array}$ & $121.48 \pm 15.5$ & $111.30 \pm 17.7$ & NS & $118.12 \pm 19.2$ & $109.88 \pm 11.23$ & NS \\
\hline $\begin{array}{l}\text { Dopamine } \\
(\mathrm{pg} / \mathrm{ml})\end{array}$ & $8.51 \pm 2.52$ & $11.74 \pm 2.2$ & NS & $8.48 \pm 2.89$ & $10.89 \pm 2.99$ & $\mathrm{NS}$ \\
\hline
\end{tabular}

\section{Effect on disease symptoms}

Symptoms of disease were assessed based on patient complaint and SCCAI score was calculated for IBD-UC patients as per standard protocol. The decrease in the SCCAI score indicates the reduction in the severity of symptoms and increase in the score indicates the augmentation in the severity of the symptom of UC. In this study reduction of 1 value in SCCAI score was considered as decrease in SCCAI score. The SCCAI score was decreased post intervention in $43.75 \%$ of the patients in the probiotic treated group which was significantly high $(\mathrm{p}<0.05)$ as compared to placebo where the decrease in SCCAI score was reported in $28.57 \%$ patients. 


\section{Effect on physical, behavioral and psychological parameters}

The enrolled subjects were assessed before and after intervention for the different physical symptoms, behavioral and psychological symptoms including stiff or tense muscles, heartburn, headache, shakiness or tremor, sleep problem, difficulty in completing work, procrastination, overwhelming, feeling of depression, trouble relaxing, nervousness, poor concentration, quick temper and restlessness. All the symptoms were evaluated based on scores, the decrease in the score indicates the reduction in the severity of symptoms and increase in the score indicates the augmentation in the severity of the symptom. In this study the complaint of muscles stiffness was reduced post intervention in $41.66 \%$ and $29.16 \%$ subjects in the treatment and placebo group respectively and the difference between the groups was significant $(\mathrm{p}<0.05)$. The complaint of heartburn was reduced post intervention in $43.75 \%$ and $31.25 \%$ subjects in the treatment and placebo group respectively and the difference between the groups was significant $(\mathrm{p}<0.05)$. The complaints of headache were reduced post intervention in $37.5 \%$ and $33.33 \%$ subjects in the probiotic and placebo group respectively with no significant difference between groups. The complaint of shakiness or tremor was reduced post intervention in $33.33 \%$ and $31.25 \%$ subjects in the treatment and placebo group with no significant difference between groups.

The complaint of sleep problem was reduced post intervention in $41.66 \%$ and 27.08 $\%$ subjects in the treatment and placebo group respectively with the difference between groups being significant $(\mathrm{p}<0.05)$. The complaint of procrastination was reduced in $31.25 \%$ subjects and $35.4 \%$ subjects in the treatment and placebo group respectively with no significant difference between groups. The complaints of difficulty in completing work or assignments was decreased post intervention in $37.5 \%$ subjects and $27.08 \%$ subjects in the probiotic treated and placebo group respectively with the difference between groups being significant $(\mathrm{p}<0.05)$. The complaints of overwhelming was reduced post intervention in $41.66 \%$ and $31.25 \%$ subjects in the treatment and placebo group respectively and the difference between placebo and treatment groups was significant $(\mathrm{p}<0.05)$. The complaints of trouble relaxing was reduced post intervention in $37.5 \%$ and $27.08 \%$ subjects in the treatment group and placebo group respectively with the difference between groups being significant ( $\mathrm{p}<0.05)$. The complaint of nervousness was reduced post intervention in 33.33 $\%$ and $29.16 \%$ subjects in the treatment and placebo group respectively with no significant difference between groups. The complaints of depression was reduced post intervention in $33.33 \%$ and $31.25 \%$ subjects in the treatment and placebo group respectively with no significant difference between groups. The complaints of poor concentration was reduced 
523 post intervention in $47.91 \%$ and $33.3 \%$ subjects in the treatment group and placebo group 524 respectively with the difference between groups being significant $(\mathrm{p}<0.05)$. The complaints 525 of quick temper was reduced post intervention in $45.83 \%$ and $39.58 \%$ subjects in the 526 treatment group and placebo group respectively with no significant difference. The 527 complaints of restlessness was reduced post intervention in $47.91 \%$ and $35.4 \%$ subjects in 528 the treatment group and placebo group respectively with the difference between groups being significant $(\mathrm{p}<0.05)$. The observed results exhibited improvement in various physical, behavioral and psychological symptoms of enrolled IBD subjects in the treatment group.

Table 2: Post intervention decrease in symptoms in the enrolled subjects for different physical, behavioral and psychological parameters

\begin{tabular}{|l|r|r|r|}
\hline $\begin{array}{l}\text { Physical , behavioural } \\
\text { and psychological } \\
\text { parameters }\end{array}$ & \multicolumn{3}{|c|}{$\begin{array}{r}\text { Post intervention decrease in symptoms } \\
\text { (\% of total subjects) }\end{array}$} \\
\hline & $\begin{array}{l}\text { Bacillus coagulans } \\
\text { Unique IS2 group }\end{array}$ & Placebo group & P value \\
\hline Muscles stiffness & $41.66 \%$ & $29.16 \%$ & $<0.05$ \\
\hline Heartburn & 43.75 & $31.25 \%$ & $<0.05$ \\
\hline Headache & $37.5 \%$ & $33.33 \%$ & NS \\
\hline Shakiness or tremor & $33.33 \%$ & $31.25 \%$ & $<0.05$ \\
\hline Sleep problem & $41.66 \%$ & $27.08 \%$ & NS \\
\hline Procrastination & $31.25 \%$ & $35.4 \%$ & $<0.05$ \\
\hline $\begin{array}{l}\text { Difficulty in completing } \\
\text { work or assignments }\end{array}$ & $37.5 \%$ & $27.08 \%$ & $<0.05$ \\
\hline Overwhelming & $41.66 \%$ & $31.25 \%$ & $<0.05$ \\
\hline Trouble relaxing & $37.5 \%$ & $27.08 \%$ & $\mathrm{NS}$ \\
\hline Nervousness & $33.33 \%$ & $29.16 \%$ & $<0.05$ \\
\hline Depression & $33.33 \%$ & $31.25 \%$ & $\mathrm{NS}$ \\
\hline Poor concentration & $47.91 \%$ & $33.3 \%$ & $<0.05$ \\
\hline Quick temper & $45.83 \%$ & $39.58 \%$ & $35.4 \%$ \\
\hline Restlessness & $47.91 \%$ & & \\
\hline
\end{tabular}

535 Safety evaluations: During and after intervention no adverse events were observed, recorded 536 and reported in the study which further established the safety of B coagulans Unique IS2. 


\section{Discussion}

Targeted microbiota intervention through probiotics and fecal microbiota transplantation are considered as effective therapeutic methods for $\operatorname{IBD}^{12,33}$. Mechanisms of action of probiotics in IBD prevention include increase in beneficial bacteria, inhibition of pathogenic bacteria, immuno-modulation, augmentation of anti-inflammatory responses and enhancement of the intestinal barrier function ${ }^{14}$. Bacillus species are high heat resistance, acid tolerance and they can survive considerably better than other probiotics in gastric conditions $^{34,35}$. B coagulans Unique IS-2 is a non-toxic commercial probiotic strain with proven safety and efficacy, long shelf life and stability at room temperature ${ }^{24}$. Whole genome sequence analysis of B. coagulans Unique IS-2 has corroborated its safety with the absence of any toxin genes ${ }^{36}$. In this study, no severe adverse event was reported, which establish the safety of $B$. coagulans Unique IS-2. Safety and therapeutic efficacy of $B$ coagulans Unique IS-2 has been proven in different disease including Irritable Bowel Syndrome in adult ${ }^{27}$ and children $^{25}$, acute-diarrhea ${ }^{26}$, abdominal pain $^{37}$, constipation $^{27}$, oral health ${ }^{38}$, bacterial vaginosis $^{26}$, anti-hypercholesterolemic effect ${ }^{22}$ and liver cirrhosis. This strain also showed anti-inflammatory ${ }^{24}$ and anti-proliferative effects ${ }^{39}$.

Post intervention, significant detection of Bacillus coagulans in the probiotic treated group demonstrating that this probiotic strain was able to survive in GI tract of IBD patients. Beneficial bacteria provide protection to host against colonization of harmful bacteria and suppress the growth of pathogens by imposing competition for shared niches and nutrients ${ }^{40}$. Results of the present study indicated that B coagulans Unique IS-2 was able to modulate the gut microbiota by increasing beneficial bacteria. Another study also reported that consumption of $B$. coagulans was capable of restoring the microbial imbalance ${ }^{41}$ and able to increase populations of Lactobacillus and Bifidobacteria ${ }^{42,43}$ possibly by consuming free oxygen in the intestine and reduces redox reactions, creating an unfavorable anaerobic and acidic environment to various pathogens ${ }^{43}$.

Results of the metagenomics analysis revealed that phylum Firmicutes, Bacteriodetes, Proteobacteria, Actinobacteria, Euryarchaeota and Verrucomicrobia were abundant in both the study group which are earlier reported dominated in Indian gut ${ }^{44,45}$. An increase in the abundance of phylum Firmicutes and Bacteriodetes was observed in post intervention sample in both groups. Decrease in abundance of phylum Proteobacteria and Actinobacteria was observed in post intervention sample in both groups. Other studies also reported decrease in Bacteroides and Firmicutes and increase in Proteobacteria and Actinobacteria in GI 
571 disease ${ }^{31,46}$. It is believed that inflammation is an oxidative state which might promote the

572

573

574

575

576

577

578

579

580

581

582

583

584

585

586

587

588

589

590

591

592

593

594

595

596

597

598

599

600

601

602

603

604 outgrowth of aerotolerant taxa such as Proteobacteria and Actinobacteria ${ }^{47}$.

The abundance of Lactobacillus, Bifidobacterium and Bacillus genera were increased in post intervention samples in treatment group and decrease in placebo group. Lactobacillus and Bifidobacteria are among the first colonizers of newborns ${ }^{48}$ and are known for their beneficial effects ${ }^{49}$. The observed high abundance of Lactobacillus, Bifidobacterium and Bacillus genera in treatment group indicated that given probiotic help the gut in restoring these beneficial bacteria. Other studies reported decreased abundance of Faecalibacterium in $\mathrm{IBD}^{50,51}$. We observed an increase in abundance of genus Faecalibacterium in post intervention samples in treatment group and decrease in placebo group which indicates the given probiotic help the gut in restoring them. Bacterial genera Lactobacillus, Bifidobacterium, and Faecalibacterium have been reported to be protective for mucosal inflammation in the host $\mathrm{t}^{1,52}$ via several mechanisms, including the up-regulation of the antiinflammatory cytokine, and down-regulation of inflammatory cytokines ${ }^{53}$. Studies reported decrease in the genera Bacteriodes in $\mathrm{IBD}^{14,54}$. We observed an increase in abundance of genus Bacteroides in post intervention samples in treatment group and decrease in placebo group which indicates that the given probiotic intervention may help to restore the genus Bacteroides in enrolled IBD patients. A decrease in the abundance of bacterial genera Dialister, Roseburia, Megasphaera was observed in post intervention samples in treatment group and increase in placebo group. A decrease in the abundance of Blautia species in the IBD patients was reported ${ }^{55}$. We observed an increase in OTUs of genus Blautia in post intervention samples in both treatment and placebo group. An increase in abundance of genus Alistipes in post intervention samples in treatment group and decrease in placebo group were observed. Gut microbiota study in the IBD patients reported that some of the Faecalibacterium, Bacteroides and Alistipes species have shown significant contribution to metabolic pathway transcription ${ }^{56}$. The abundance of some of bacterial taxon was low but these may play important role in gut function as reported earlier ${ }^{57}$.

The improper host immune response against GI microbiota is considered to be the main reason in causing severe inflammation ${ }^{40}$. Studies have reported the changes in the serum levels of anti- inflammatory cytokine (IL-10) and pro- inflammatory cytokines (IL-6, IL-12, TNF- $\alpha$, INF- $\gamma$ ) in GI disorders ${ }^{58}$. However, the serum cytokine profiling of IBD patients remains less reported. In the present study significant increase was observed in IL-10 levels in treatment group which indicated that the probiotic strain was able to increase the secretion of IL-10 in IBD patients in the treatment group. Other studies have reported the association of 
605 IBD patients with anti-inflammatory cytokines IL-10 $0^{59}$ and IL-10 secretion increased during 606 disease recovery in IBD patients ${ }^{60}$. It is also reported that inactivation of IL-10 leads to 607 increased release of pro-inflammatory cytokines ${ }^{61}$.

608 In the present study we observed the significant decrease in IL6 ( $\mathrm{p}<0.05$ ), IL17 (p $<0.05)$, IL23 ( $\mathrm{p}<0.05)$, andIL-1 $\beta$ ( $\mathrm{p}<0.05)$, TNF- $\alpha$ in treatment group which indicated the probiotic intervention was able to modulate the secretion of pro-inflammatory cytokines. Previous studies have reported increased expression of IL-6 may be an intestinal inflammatory mediator of IBD $^{64}$. Studies have reported that the expression of IL-6 was predominantly detected in IBD and an association between serum levels of IL-6 and disease activity $^{62}$. A study reported IL6 in active UC $26+/-10 \mathrm{pg} / \mathrm{ml}$ and in inactive UC $<10 \mathrm{pg} / \mathrm{ml}^{63}$ and this suggested that increased expression of IL-6 may be an intestinal inflammatory mediator of IBD. IL-17 induces the production of many other pro-inflammatory cytokines, including IL-6, TNF- $\alpha$, and IL-1 $\beta$, which leads to localizing and amplifying inflammation. IL-17 was reported to be increased in intestinal tissue and serum of IBD patients ${ }^{64,65}$. IL-1 $\beta$ is a pro-inflammatory cytokines play important role in the inflammation in patients with IBD and an elevation in IL-1 $\beta$ levels are associated with increased disease severity ${ }^{66-68}$. Studies reported that the improper level of serotonin and dopamine increases the severity of IBD $^{69}$. Serum serotonin and dopamine were also evaluated in this study but no significant change was observed in both groups.

Results of the study indicated that $B$ coagulans Unique IS-2 along with SMT was able to reduce the severity of symptom and improve physical and psychological parameters in IBD patient in the treatment group. These results are similar to another study which reported that a probiotic mixture (VSL\#3) reduced the expression of inflammatory cytokines and the severity of disease in UC patients ${ }^{70}$. A meta-analysis also reported that probiotics can benefit IBD treatment during combined use of probiotics and standard therapy ${ }^{71}$. A study with $B$. coagulans Unique IS-2 in children with functional abdominal pain indicated reduction of abdominal pain in the probiotic treated group ${ }^{37}$. Another study reported B. coagulans Unique IS-2 was effective in the treatment of IBS with a significant decrease in the intensity of pain

633 in the probiotic treated group ${ }^{25}$. Probiotics can reduce inflammation and disease symptoms 634 by modulation of the mucosal immune system, increased intestinal barrier function, 635 competitive prohibition of pathogens, production of antimicrobial factors ${ }^{72}$ amplification of 636 the intestinal tight junctions to stabilize the permeability, normalize bowel movements and 637 reduce visceral hypersensitivity ${ }^{73-75}$. 


\section{Summary and Conclusion}

The results of the study showed that the $B$ coagulans Unique IS-2 is able to survive in GI tract of IBD patients. B. coagulans Unique IS-2 was able to enhance bacterial genera Lactobacillus, Bifidobacterium, Faecalibacterium, Bacteroides, Megamonas, Lachnospira, Blautia and Alistipes in post intervention samples in the treatment group. A decrease in bacterial genera Sutterella, Dialister, Roseburia and Megasphaera was observed in post intervention samples in the treatment group. Variable alterations were also observed in the abundance of different bacterial taxon including phylum, class, order, family, and genus in the post intervention sample of the treatment group. B coagulans Unique IS-2 was able to modulate the secretion of serum cytokines in IBD patients. The level of IL-10 was increased significantly post intervention in treatment group. The secretion of cytokines, IL-6, IL-1 $\beta$, TNF- $\alpha$, IL -17 and IL -23 were variably decreased post intervention in the treatment group. No significant change in serum serotonin and dopamine was observed in both treatment and placebo groups. A reduction in the severity of symptoms of disease and significant improvement in the physical and psychological parameter were observed post intervention in enrolled subjects in the treatment group. Observed results demonstrated that $B$ coagulans Unique IS2 showed beneficial effect in IBD-UC patients when administered along with standard medical treatment (SMT). Study was registered with Clinical Trials Registry India (CTRI) - (CTRI registration No.- CTRI/2019/11/022087).

Conflict of interest statement: RSM and JN are employed by manufacturer of probiotics (Unique Biotech Ltd) and they wish to state that the study was conducted independently with no intervention on their part during the study. All other authors declare no conflict of interest. Author Contribution: VDB- Recruited the subjects, carried out experiments, analyzed data and wrote final manuscript, DD - carried out experiments and analyzed the data, PS - carried out experiments and analyzed the data. SK clinical assessment and monitoring of subjects, RSM, JN - drafted study proposal and manuscript, VA- clinical assessment and monitoring of subjects, designed the trial, supervised the study. RC -conceptualizes the study, finalized study proposal, designed the trial and supervised the study. All the authors read and revised the manuscript and approve the final manuscript.

Acknowledgement: Authors acknowledge the financial support from Unique Biotech Ltd, Hyderabad, India. Help of Mr. Surender Singh (Laboratory Technician) in microbial culture media and reagent preparation is also acknowledged. 


\section{References}

1. Acharyya BC. Incidence of Inflammatory Bowel Disease in a Tertiary Centre of Eastern India. ARGH; 9. Epub ahead of print 26 July 2018. DOI: 10.19080/ARGH.2018.09.555774.

2. Kedia S, Ahuja V. Is the emergence of inflammatory bowel disease a prime example of 'the third epidemiological transition'? Indian J Gastroenterol 2018; 37: 183-185.

3. Singh P, Ananthakrishnan A, Ahuja V. Pivot to Asia: inflammatory bowel disease burden. Intest Res 2017; 15: 138-141.

4. Kedia S, Ahuja V. Epidemiology of Inflammatory Bowel Disease in India: The Great Shift East. Inflamm Intest Dis 2017; 2: 102-115.

5. Kim YS, Jung S-A, Lee K-M, et al. Impact of inflammatory bowel disease on daily life: an online survey by the Korean Association for the Study of Intestinal Diseases. Intest Res 2017; 15: 338-344.

6. Sigall-Boneh R, Levine A, Lomer M, et al. Research Gaps in Diet and Nutrition in Inflammatory Bowel Disease. A Topical Review by D-ECCO Working Group [Dietitians of ECCO]. J Crohns Colitis 2017; 11: 1407-1419.

7. Ko JK, Auyeung KK. Inflammatory bowel disease: etiology, pathogenesis and current therapy. Curr Pharm Des 2014; 20: 1082-1096.

8. Maunder RG, Levenstein S. The role of stress in the development and clinical course of inflammatory bowel disease: epidemiological evidence. Curr Mol Med 2008; 8: 247252.

9. Kim DH, Cheon JH. Pathogenesis of Inflammatory Bowel Disease and Recent Advances in Biologic Therapies. Immune Netw 2017; 17: 25-40.

10. Nishida A, Inoue R, Inatomi $\mathrm{O}$, et al. Gut microbiota in the pathogenesis of inflammatory bowel disease. Clin J Gastroenterol 2018; 11: 1-10.

11. Zuo $\mathrm{T}, \mathrm{Ng} \mathrm{SC}$. The Gut Microbiota in the Pathogenesis and Therapeutics of Inflammatory Bowel Disease. Front Microbiol 2018; 9: 2247.

12. Dong L-N, Wang M, Guo J, et al. Role of intestinal microbiota and metabolites in inflammatory bowel disease. Chin Med J (Engl) 2019; 132: 1610-1614.

13. Sun Y, Li L, Xia Y, et al. The gut microbiota heterogeneity and assembly changes associated with the IBD. Sci Rep 2019; 9: 440.

14. Khan I, Ullah N, Zha L, et al. Alteration of Gut Microbiota in Inflammatory Bowel Disease (IBD): Cause or Consequence? IBD Treatment Targeting the Gut Microbiome. Pathogens 2019; 8: 126.

15. Manocha M, Khan WI. Serotonin and GI Disorders: An Update on Clinical and Experimental Studies. Clin Transl Gastroenterol 2012; 3: e13.

16. O'Mahony SM, Clarke G, Borre YE, et al. Serotonin, tryptophan metabolism and the brain-gut-microbiome axis. Behav Brain Res 2015; 277: 32-48.

17. Tolstanova G, Deng X, Ahluwalia A, et al. Role of Dopamine and D2 Dopamine Receptor in the Pathogenesis of Inflammatory Bowel Disease. Dig Dis Sci 2015; 60: 2963-2975.

18. Rhee SH, Pothoulakis C, Mayer EA. Principles and clinical implications of the brain-gutenteric microbiota axis. Nat Rev Gastroenterol Hepatol 2009; 6: 306-314. 
19. Reber SO. Stress and animal models of inflammatory bowel disease-An update on the role of the hypothalamo-pituitary-adrenal axis. Psychoneuroendocrinology 2012; 37: 119.

20. Sudha M, Chauhan P, Dixit K, et al. Molecular Typing and Probiotic Attributes of a New Strain of Bacillus coagulans - Unique IS-2: a Potential Biotherapeutic Agent. undefined, https://www.semanticscholar.org/paper/Molecular-Typing-and-Probiotic-Attributes-of-aNew-Sudha-Chauhan/f8bbf999bc57cb022d282e9ad13442f877914db7 (2010, accessed 28 July 2021).

21. Konuray G, Erginkaya Z. Potential Use of Bacillus coagulans in the Food Industry. Foods 2018; 7: 92.

22. Ratna Sudha M, Radkar N, Maurya A. Effect of supplementation of probiotic bacillus coagulans unique IS-2 (ATCC PAT-11748) on hypercholesterolemic subjects: A clinical study. International Journal of Probiotics and Prebiotics 2011; 6: 89-94.

23. Ratna Sudha M, Bhonagiri S. Efficacy of Bacillus coagulans strain unique IS-2 in the treatment of patients with acute diarrhea. International Journal of Probiotics and Prebiotics 2012; 7: 33-37.

24. Sudha RM, Sunita M, Sekhar BM. Safety studies of bacillus coagulans unique IS-2 in rats: Morphological, biochemical and clinical evaluations. International Journal of Probiotics \& Prebiotics 2016; 11: 43+.

25. Sudha MR, Jayanthi N, Aasin M, et al. Efficacy of Bacillus coagulans Unique IS2 in treatment of irritable bowel syndrome in children: a double blind, randomised placebo controlled study. Benef Microbes 2018; 9: 563-572.

26. Ratna Sudha M, Yelikar KA, Deshpande S. Clinical Study of Bacilluscoagulans Unique IS-2 (ATCC PTA-11748) in the Treatment of Patients with Bacterial Vaginosis. Indian J Microbiol 2012; 52: 396-399.

27. Madempudi RS, Neelamraju J, Ahire JJ, et al. Bacillus coagulans Unique IS2 in Constipation: A Double-Blind, Placebo-Controlled Study. Probiotics Antimicrob Proteins 2020; 12: 335-342.

28. Walmsley RS, Ayres RC, Pounder RE, et al. A simple clinical colitis activity index. Gut 1998; 43: 29-32.

29. Derogatis LR, Lipman RS, Rickels K, et al. The Hopkins Symptom Checklist (HSCL). Psychological Measurements in Psychopharmacology 1974; 7: 79-110.

30. Kleppang AL, Hagquist C. The psychometric properties of the Hopkins Symptom Checklist-10: a Rasch analysis based on adolescent data from Norway. Fam Pract 2016; 33: 740-745.

31. Bamola VD, Ghosh A, Kapardar RK, et al. Gut microbial diversity in health and disease: experience of healthy Indian subjects, and colon carcinoma and inflammatory bowel disease patients. Microb Ecol Health Dis 2017; 28: 1322447.

32. Ahmed N, Daniel B, Varghese J, et al. Oropharyngeal microbiome of an HIV-positive patient. Microbial Pathogenesis 2020; 139: 103805.

33. Hudson LE, Anderson SE, Corbett AH, et al. Gleaning Insights from Fecal Microbiota Transplantation and Probiotic Studies for the Rational Design of Combination Microbial Therapies. Clin Microbiol Rev 2017; 30: 191-231. 
776

777

778

779

780

781

782

783

784

785

786

787

788

789

790

791

792

793

794

795

796

797

798

799
34. Hyronimus B, Le Marrec C, Sassi AH, et al. Acid and bile tolerance of spore-forming lactic acid bacteria. Int J Food Microbiol 2000; 61: 193-197.

35. Keller D, Verbruggen S, Cash H, et al. Spores of Bacillus coagulans GBI-30, 6086 show high germination, survival and enzyme activity in a dynamic, computer-controlled in vitro model of the gastrointestinal tract. Benef Microbes 2019; 10: 77-87.

36. Upadrasta A, Pitta S, Madempudi RS. Draft Genome Sequence of the Spore-Forming Probiotic Strain Bacillus coagulans Unique IS-2. Genome Announc 2016; 4: e00225-16.

37. Saneian H, Pourmoghaddas Z, Roohafza H, et al. Synbiotic containing Bacillus coagulans and fructo-oligosaccharides for functional abdominal pain in children. Gastroenterol Hepatol Bed Bench 2015; 8: 56-65.

38. K MJ, Shenoy N, Talwar A, et al. Clinical effect of pro-biotic containing Bacillus coagulans on plaque induced gingivitis $\square$ : A randomised clinical pilot study. Journal of Health and Allied Sciences NU 2017; 07: 007-012.

39. Madempudi RS, Kalle AM. Antiproliferative Effects of Bacillus coagulans Unique IS2 in Colon Cancer Cells. Nutr Cancer 2017; 69: 1062-1068.

40. Kho ZY, Lal SK. The Human Gut Microbiome - A Potential Controller of Wellness and Disease. Front Microbiol 2018; 9: 1835.

41. Hempel S, Newberry SJ, Maher AR, et al. Probiotics for the prevention and treatment of antibiotic-associated diarrhea: a systematic review and meta-analysis. JAMA 2012; 307: 1959-1969.

42. Ara K, Meguro S, Hase T, et al. Effect of Spore-bearing Lactic Acid-forming Bacteria ( Bacillus coagulans SANK 70258) Administration on the Intestinal Environment, Defecation Frequency, Fecal Characteristics and Dermal Characteristics in Humans and Rats. Microbial Ecology in Health and Disease 2002; 14: 4-13.

43. Cao J, Yu Z, Liu W, et al. Probiotic characteristics of Bacillus coagulans and associated implications for human health and diseases. Journal of Functional Foods 2020; 64: 103643.

44. Jain A, Li XH, Chen WN. Similarities and differences in gut microbiome composition correlate with dietary patterns of Indian and Chinese adults. AMB Expr 2018; 8: 104.

45. Gupta A, Dhakan DB, Maji A, et al. Association of Flavonifractor plautii, a FlavonoidDegrading Bacterium, with the Gut Microbiome of Colorectal Cancer Patients in India. mSystems; 4: e00438-19.

46. Ryan FJ, Ahern AM, Fitzgerald RS, et al. Colonic microbiota is associated with inflammation and host epigenomic alterations in inflammatory bowel disease. Nat Commun 2020; 11: 1512.

47. Ni J, Wu GD, Albenberg L, et al. Gut microbiota and IBD: causation or correlation? Nat Rev Gastroenterol Hepatol 2017; 14: 573-584.

48. Feng Q, Liang S, Jia H, et al. Gut microbiome development along the colorectal adenoma-carcinoma sequence. Nat Commun 2015; 6: 6528.

49. Flint HJ, Scott KP, Duncan SH, et al. Microbial degradation of complex carbohydrates in the gut. Gut Microbes 2012; 3: 289-306.

50. Becker C, Neurath MF, Wirtz S. The Intestinal Microbiota in Inflammatory Bowel Disease. ILAR J 2015; 56: 192-204. 
51. Wang W, Chen L, Zhou R, et al. Increased proportions of Bifidobacterium and the Lactobacillus group and loss of butyrate-producing bacteria in inflammatory bowel disease. J Clin Microbiol 2014; 52: 398-406.

52. Joossens M, Huys G, Cnockaert M, et al. Dysbiosis of the faecal microbiota in patients with Crohn's disease and their unaffected relatives. Gut 2011; 60: 631-637.

53. Sokol H, Pigneur B, Watterlot L, et al. Faecalibacterium prausnitzii is an antiinflammatory commensal bacterium identified by gut microbiota analysis of Crohn disease patients. Proc Natl Acad Sci U S A 2008; 105: 16731-16736.

54. Nemoto H, Kataoka K, Ishikawa $H$, et al. Reduced diversity and imbalance of fecal microbiota in patients with ulcerative colitis. Dig Dis Sci 2012; 57: 2955-2964.

55. Gevers D, Kugathasan S, Denson LA, et al. The treatment-naive microbiome in newonset Crohn's disease. Cell Host Microbe 2014; 15: 382-392.

56. Schirmer M, Franzosa EA, Lloyd-Price J, et al. Dynamics of metatranscription in the inflammatory bowel disease gut microbiome. Nat Microbiol 2018; 3: 337-346.

57. Arumugam M, Raes J, Pelletier E, et al. Enterotypes of the human gut microbiome. Nature 2011; 473: 174-180.

58. Bennet SMP, Polster A, Törnblom H, et al. Global Cytokine Profiles and Association With Clinical Characteristics in Patients With Irritable Bowel Syndrome. Am J Gastroenterol 2016; 111: 1165-1176.

59. Braat H, Rottiers P, Hommes DW, et al. A phase I trial with transgenic bacteria expressing interleukin-10 in Crohn's disease. Clin Gastroenterol Hepatol 2006; 4: 754 759.

60. Mitsuyama K, Tomiyasu N, Takaki K, et al. Interleukin-10 in the pathophysiology of inflammatory bowel disease: increased serum concentrations during the recovery phase. Mediators Inflamm 2006; 2006: 26875.

61. Ma X, Yan W, Zheng H, et al. Regulation of IL-10 and IL-12 production and function in macrophages and dendritic cells. F1000Res 2015; 4: F1000 Faculty Rev-1465.

62. Mavropoulou E, Mechie N-C, Knoop R, et al. Association of serum interleukin-6 and soluble interleukin-2-receptor levels with disease activity status in patients with inflammatory bowel disease: A prospective observational study. PLOS ONE 2020; 15 : e0233811.

63. Holtkamp W, Stollberg T, Reis HE. Serum interleukin-6 is related to disease activity but not disease specificity in inflammatory bowel disease. J Clin Gastroenterol 1995; 20: 123-126.

64. Kuwabara T, Ishikawa F, Kondo M, et al. The Role of IL-17 and Related Cytokines in Inflammatory Autoimmune Diseases. Mediators of Inflammation 2017; 2017 : e3908061.

65. Nemeth ZH, Bogdanovski DA, Barratt-Stopper P, et al. Crohn's Disease and Ulcerative Colitis Show Unique Cytokine Profiles. Cureus 2017; 9: e1177.

66. Mao L, Kitani A, Strober W, et al. The Role of NLRP3 and IL-1 $\beta$ in the Pathogenesis of Inflammatory Bowel Disease. Front Immunol 2018; 9: 2566.

67. Coccia M, Harrison OJ, Schiering C, et al. IL-1 $\beta$ mediates chronic intestinal inflammation by promoting the accumulation of IL-17A secreting innate lymphoid cells and CD4(+) Th17 cells. J Exp Med 2012; 209: 1595-1609. 
68. Ligumsky M, Simon PL, Karmeli F, et al. Role of interleukin 1 in inflammatory bowel disease--enhanced production during active disease. Gut 1990; 31: 686-689.

69. Marcus MM, Jardemark K, Malmerfelt A, et al. Augmentation by escitalopram, but not citalopram or R-citalopram, of the effects of low-dose risperidone: behavioral, biochemical, and electrophysiological evidence. Synapse 2012; 66: 277-290.

70. Tursi A, Brandimarte G, Papa A, et al. Treatment of relapsing mild-to-moderate ulcerative colitis with the probiotic VSL\#3 as adjunctive to a standard pharmaceutical treatment: a double-blind, randomized, placebo-controlled study. Am J Gastroenterol 2010; 105: 2218-2227.

71. Ganji-Arjenaki M, Rafieian-Kopaei M. Probiotics are a good choice in remission of inflammatory bowel diseases: A meta analysis and systematic review. Journal of Cellular Physiology 2018; 233: 2091-2103.

72. Schlee M, Harder J, Köten B, et al. Probiotic lactobacilli and VSL\#3 induce enterocyte beta-defensin 2. Clin Exp Immunol 2008; 151: 528-535.

73. Didari T, Mozaffari S, Nikfar S, et al. Effectiveness of probiotics in irritable bowel syndrome: Updated systematic review with meta-analysis. World J Gastroenterol 2015; 21: 3072-3084.

74. Gareau MG, Sherman PM, Walker WA. Probiotics and the gut microbiota in intestinal health and disease. Nat Rev Gastroenterol Hepatol 2010; 7: 503-514.

75. Korterink JJ, Ockeloen L, Benninga MA, et al. Probiotics for childhood functional gastrointestinal disorders: a systematic review and meta-analysis. Acta Paediatr 2014; 103: 365-372. 